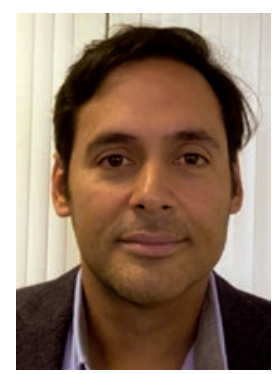

\title{
A debate: MitraClip. Perspectiva del experto en insuficiencia cardiaca
}

\section{Debate: MitraClip. The heart failure expert perspective}

\author{
Julio Núñez ${ }^{\mathrm{a}, \mathrm{b}}$,* \\ a Servicio de Cardiología, Hospital Clínico Universitario de Valencia, INCLIVA, Universidad de Valencia, Valencia, España \\ ${ }^{\mathrm{b}}$ CIBER de Enfermedades Cardiovasculares (CIBERCV), España
}

VÉASE CONTENIDO RELACIONADO:

https://doi.org/10.24875/RECIC.M19000008

Pregunta: Sin duda, lo más relevante de 2018 en el ámbito de la cardiología intervencionista fueron los tan esperados resultados de los ensayos con MitraClip (Abbott Laboratories, Abbott Park, Illinois, Estados Unidos): el MITRA-FR (Multicentre Study of Percutaneous Mitral Valve Repair MitraClip Device in Patients With Severe Secondary Mitral Regurgitation) en el congreso de la Sociedad Europea de Cardiología y el COAPT /Cardiovascular Outcomes Assessment of the MitraClip Percutaneous Therapy for Heart Failure Patients With Functional Mitral Regurgitation) en el congreso Transcatheter Cardiovascular Therapeutics (TCT). Se ha comentado mucho sobre los dos ensayos. ¿Podría hablarnos de las diferencias básicas que explican los diferentes resultados observados en ellos?

RESPUESTA: En efecto, la publicación de ambos estudios ha puesto en el candelero a la insuficiencia mitral (IM) como diana terapéutica en los pacientes con insuficiencia cardiaca y fracción de eyección reducida (ICFER). En el ensayo COAPT se aleatorizaron 614 pacientes con ICFER (fracción de eyección del ventrículo izquierdo entre el 20 y el 50\%) e IM moderada-grave tratados con terapia medica óptima para seguir dos estrategias de tratamiento (1:1): a) tratamiento médico óptimo, o b) tratamiento médico óptimo más implante percutáneo de dispositivo MitraClip ${ }^{1}$. A los 2 años se observó una reducción del $47 \%$ en el riesgo del objetivo primario de eficacia (hospitalizaciones por insuficiencia cardiaca) en el grupo de intervención. Del mismo modo, se constató una reducción significativa de la mortalidad por todas las causas (hazard ratio $=0,62$; intervalo de confianza del 95\%, 0,46-0,82; $p<0,001)$ y de otros objetivos secundarios ${ }^{1}$. En cuanto al objetivo primario de seguridad, se confirmó un número relativamente bajo de complicaciones relacionadas con la técnica a los 12 meses de la intervención. El número necesario de pacientes a tratar fue de 3,1 para evitar una hospitalización y de 5,7 para evitar una muerte. Estos datos de eficacia clínica son muy superiores los observados con la inmensa mayoría de los fármacos de contrastada evidencia en la ICFER, y comparables a los de otras medidas como la administración de antibióticos en enfermedades infecciosas o el implante percutáneo de válvula aórtica en la estenosis aórtica grave.
Sin embargo, este cuento de hadas se ve ensombrecido por la publicación casi simultánea (aproximadamente un mes antes) de los resultados del estudio aleatorizado abierto MITRA-FR, en el que se evaluaron la eficacia y la seguridad del dispositivo MitraClip en 307 sujetos con ICFER e IM secundaria moderadagrave $^{2}$. En este ensayo, en comparación con el tratamiento médico estándar, MitraClip no se asoció a una mejoría en el riesgo del objetivo combinado de muerte por todas las causas o ingreso por insuficiencia cardiaca a 12 meses $^{2}$.

A priori, ambos estudios incluyeron pacientes con ICFER e IM secundaria moderada-grave. Además, es importante apuntar que las características basales de los sujetos incluidos en ambos estudios son similares en importantes características clínicas y de riesgo. Sin embargo, hay que destacar ciertas diferencias ecocardiográficas, resultados de la intervención, tratamiento médico y tiempo de seguimiento, que podrían estar detrás de tan discordantes resultados:

- Diferencias ecocardiográficas: este es uno de los puntos clave al interpretar las divergencias entre ambos estudios. De hecho, el área del orificio regurgitante efectivo de la IM fue inferior en el MITRA-FR que en el COAPT $\left(31 \pm 10 \mathrm{~mm}^{2}\right.$ frente a $41 \pm 15 \mathrm{~mm}^{2}$ ). Del mismo modo, los volúmenes telediastólicos del ventrículo izquierdo fueron mayores en el MITRA-FR que en el COAPT $\left(135 \pm 35 \mathrm{ml} / \mathrm{m}^{2}\right.$ frente a $\left.101 \pm 34 \mathrm{ml} / \mathrm{m}^{2}\right)$. Estos dos hechos apuntan a que los pacientes que más podrían beneficiarse de esta intervención son aquellos con mayor grado de disfunción valvular y menor dilatación ventricular izquierda; en otras palabras, con predominio de la valvulopatía sobre la enfermedad ventricular izquierda.

- Diferencias en los resultados obtenidos con la intervención: en el estudio COAPT destaca que la frecuencia de complicaciones periprocedimiento o de resultado subóptimo inmediatamente y a los 12 meses tras la intervención fue muy inferior que en el estudio MITRA-FR. Por ejemplo, en el estudio COAPT solo un $5 \%$ de los pacientes mostraron una IM de

\footnotetext{
* Autor para correspondencia: Servicio de Cardiología, Hospital Clínico Universitario de Valencia, Avda. Blasco Ibáñez 17, 46010 Valencia, España. Correo electrónico:yulnunez@gmail.com (J. Núñez). 
grado $\geq$ III a los 12 meses de seguimiento ${ }^{1}$, mientras que este porcentaje llegó al $17 \%$ en el estudio MITRA-FR ${ }^{2}$. Este hecho sugiere un papel crucial de la experiencia y la pericia del equipo intervencionista en el implante. En este sentido, y aunque en ambos estudios el número de centros participantes fue elevado, sería muy pertinente conocer los resultados de eficacia y de seguridad según el volumen de implantes realizados en cada centro.

- Diferencias en el abordaje farmacológico: en el momento basal cabe destacar la alta proporción de pacientes tratados con fármacos de contrastada evidencia en la ICFER en ambos estudios. Sin embargo, en el estudio COAPT la proporción de pacientes tratados con inhibidores de la enzima de conversión de la angiotensina, antagonistas de los receptores de la angiotensina II o sacubitril/valsartán al inicio fue ligeramente superior en el grupo de intervención. Llama la atención que estas diferencias fueron aumentando a lo largo del seguimiento $y$, en efecto, a los 12 meses un 13,4\% más de pacientes en el grupo de intervención recibían inhibidores de la enzima de conversión de la angiotensina, antagonistas de los receptores de angiotensina II o sacubitril/valsartán. Del mismo modo, a los 12 meses de seguimiento, en el grupo de intervención el porcentaje de pacientes tratados con bloqueadores beta fue un $6,6 \%$ mayor. Dado que se trata de un estudio abierto, este hecho podría sugerir cierto sesgo de realización (el conocimiento del grupo al que están asignados los participantes en el ensayo repercute en diferencias sistemáticas en cuidados y tratamientos entre ambos grupos de intervención) y de detección (el conocimiento del grupo de tratamiento afecta las decisiones clínicas importantes, como puede ser la necesidad de hospitalizar o no a un paciente). Otro punto relativo al tratamiento médico que vale la pena comentar es la falta de información sobre las dosis absolutas de los fármacos empleados para la ICFER. Es sorprendente que en el estudio COAPT, en el que se requería tratamiento médico óptimo para la inclusión de los pacientes, no se haga referencia a las dosis farmacológicas absolutas, al inicio ni durante la evolución, de los principales grupos farmacológicos (solo se mencionan cambios relativos de dosis). Del mismo modo, tampoco hay información referente a la intensidad del seguimiento clínico que se llevó a cabo, ni sobre la administración de tratamiento diurético intravenoso de manera ambulatoria (muchos ingresos hospitalarios pueden evitarse con un seguimiento estrecho y la intensificación del tratamiento diurético). Todos estos aspectos podrían, al margen de la intervención, inclinar la balanza de resultados a favor de uno de los dos grupos de tratamiento, en particularmente con respecto al riesgo de hospitalizaciones.

- Diferentes tiempos de evaluación: es importante apuntar que los resultados relativos a la eficacia en el MITRA-FR son a 12 meses de la intervención, mientras que los del COAPT son a 24 meses. Si disecamos los resultados de ambos estudios de acuerdo con el tiempo, observamos que el mayor beneficio del MitraClip en el estudio COAPT se observó a partir de los 12 meses de seguimiento. En efecto, en el estudio COAPT, la mortalidad por todas las causas a 1 año no difirió entre ambas estrategias de tratamiento (hazard ratio $=0,81$; intervalo de confianza del 95\%, 0,57-1,15). También hay que destacar que en el estudio COAPT el seguimiento fue más largo en el grupo de intervención, no solo por la mayor mortalidad en el grupo control, sino por el número más alto de pacientes retirados del estudio en el grupo de tratamiento médico (sesgo de desgaste).

P.: Se han destacado mucho las virtudes del COAPT y las limitaciones del MITRA-FR, ¿pero cuáles serían para usted los aspectos positivos del MITRA-FR y los aspectos críticos del COAPT?
R.: En primer lugar, cabe destacar que ambos son estudios aleatorizados abiertos no comparados con placebo. Desde una perspectiva general, hay que poner en relieve las limitaciones y los sesgos a los que pueden verse sometidos este tipo de estudios frente a los ensayos a doble ciego. Además, la falta de «procedimiento simulado» en ambos no permite estimar la trascendencia del efecto placebo en este escenario. En el campo del intervencionismo tenemos como ejemplo más reciente la importante y dramática diferencia de resultados obtenidos entre los estudios abiertos y a doble ciego con un "procedimiento simulado» que se observaron para la denervación renal.

Los principales aspectos críticos del COAPT se han mencionado en el punto anterior, pero además destaca que los criterios de selección de este estudio no explican con detalle la rigurosa selección de pacientes que parece haberse llevado a cabo (de los 1.576 pacientes preseleccionados, solo se aleatorizaron 614). De hecho, la causa más frecuente para ser no elegible tras la preselección $(\mathrm{n}=419)$ fue tan vaga como "preselección incompleta u otros».

De modo general, destacaría que MITRA-FR nos ha puesto los pies en la Tierra, mostrándonos que el intervencionismo sobre la válvula mitral parece no funcionar en todos los pacientes con ICFER e IM secundaria de grado III-IV. Una vez más se ha evidenciado que necesitamos avanzar en medicina de precisión, buscando herramientas que nos permitan comprender mejor la heterogeneidad de este complejo síndrome, para así elegir los tratamientos adecuados para cada caso concreto.

P.: ¿Cómo se complementan los dos estudios para definir el candidato idóneo para la técnica?

R.: Como ya hemos comentado, creo que los dos estudios permiten entrever que el sujeto que más podría beneficiarse de esta técnica es aquel con tratamiento médico optimizado que persiste sintomático y en el que, en situación de estabilidad clínica, se constata IM más grave (por ejemplo, área del orificio de regurgitación efectivo $>30 \mathrm{~mm}^{2}$ ) con ventrículo izquierdo aún no muy dilatado. No obstante, la adecuación de este perfil debe confirmarse en futuros estudios.

P.: Una de las diferencias clave entre los ensayos fue el grado de optimización del tratamiento médico alcanzado antes de la aleatorización, lo que influyó en la frecuencia y la magnitud de los cambios terapéuticos realizados durante el seguimiento en ambos estudios. ¿Cree que es posible garantizar el grado de adecuación o maximización del estudio COAPT en la práctica clínica habitual? ¿Cómo podría lograrse desde un punto de vista organizativo?

R.: Este es un punto crucial. Aunque en ambos estudios el tratamiento antes de la aleatorización podría considerarse adecuado, la optimización del tratamiento médico fue más liberal en el MITRA-FR. Desafortunadamente, como ya señalamos antes, hay muchos datos sobre las dosis absolutas, su titulación a lo largo del tiempo y la intensidad del seguimiento que no están disponibles. Sin duda, su conocimiento y análisis nos podrían hacer entender la influencia de la adecuación del tratamiento médico en los resultados obtenidos en cada estudio. Lo que parece claro es que la optimización del tratamiento médico debería ser una condición previa para considerar el MitraClip como alternativa terapéutica.

En resumen, basándonos en los resultados de ambos estudios, parece claro que la obtención de desenlaces clínicos satisfactorios pasa por una cuidadosa selección de los pacientes, evitando la generalización del tratamiento percutáneo de la IM secundaria en la mayoría de los pacientes con ICFER. En estos momentos, corremos el riesgo de que una selección inadecuada de candidatos pueda conducir a resultados cuestionables. Por un lado, un afán 
desmedido de algunos equipos intervencionistas que busquen afianzar o iniciar su andadura en el tratamiento percutáneo de la IM, y por otro lado algunos clínicos buscando nuevas alternativas terapéuticas en pacientes muy avanzados y con mala evolución clínica, podrían amenazar la implantación exitosa de programas de intervencionismo percutáneo sobre la IM en pacientes con ICFER.

Afortunadamente, pronto se publicarán nuevos resultados que inclinen la balanza y que, finalmente, nos permitan una selección más precisa de los candidatos a intervencionismo con MitraClip. Mientras tanto, los profesionales (especialistas en imagen, especialistas en insuficiencia cardiaca e intervencionistas) deberíamos ponernos de acuerdo y sentar las bases para la implantación de equipos y programas que, de manera juiciosa y prudente, evalúen de manera individual caso a caso. Es en estas situaciones en las que cobra plena vigencia y claridad el refranero español cuando dice «vísteme despacio que tengo prisa».

\section{FINANCIACIÓN}

Este trabajo se realizó con financiación recibida desde el CIBERCV 16/11/00420.

\section{CONFLICTO DE INTERESES}

J. Núñez declara haber recibido honorarios de Abbott por presentaciones orales.

\section{BIBLIOGRAFÍA}

1. Stone GW, Lindenfeld J, Abraham WT, et al.; for the COAPT Investigators. Transcatheter Mitral-Valve Repair in Patients with Heart Failure. $N$ Engl $J$ Med. 2018;379:2307-2318.

2. Obadia JF, Messika-Zeitoun D, Leurent G, et al.; for the MITRA-FR Investigators. Percutaneous Repair or Medical Treatment for Secondary Mitral Regurgitation. N Engl J Med. 2018;379:2297-2306. 